\title{
A Finite Element Modeling Study on the Fingertip Deformation under Pressure Stimulation
}

\author{
Huiling Chen ${ }^{1}$, Liguo Shuai ${ }^{1}$, Lei zhang ${ }^{2}$, Mei Miao ${ }^{3}$ \\ ${ }^{1}$ School of Mechanical Engineering, Southeast University, Nanjing 211189, China \\ ${ }^{2}$ School of Mechanical Engineering, Nantong University, Nantong 226019, China \\ ${ }^{3}$ Institute of Mathematics and Computer, Jiangsu Second Normal University, Nanjing 210013, China
}

\begin{abstract}
Pressure stimulus causes skin deformation and tactile sensation on the fingertip. Both theoretical approach and experimental technique may be used to investigate the relationship between the deformation and the sensation. Building an appropriate skin model is the most important step for further theoretical and experimental analysis. In this paper, a two dimensional (2D) fingertip biomechanical model employing finite element (FE) method is proposed based on the physiological structure of skin. With biomechanical and electrophysiological simulations, the predicted distributions of the strain energy density (SED) and the stress/strain are obtained. The relation between the predicted biomechanical responses of the subcutaneous tissue and the discharged rate reported in the literatures are investigated. The results show that the soft tissues of fingertips are very sensitive to the external stimulus, and the spatial distribution characteristics of SED within soft tissues can explain the evoked charging rate of mechanoreceptors effectively. The simulation data of the proposed FE model is highly consistent with the verified data.
\end{abstract}

\section{Introduction}

The touch feeling on skin mainly includes pressure, vibration and temperature perception. When someone touches an object with his finger, his skin will be deformed under force, and the inside receptors may also have a subtle deformation, which will trigger a tactile related electrical impulse. This signal is transferred via the peripheral nerve system and finally transmitted to the brain to tell the subject that there is a touch action on the fingertip. The nerve impulse frequency is associated with both the mechanical environment such as stress/strain and the SED around the receptors [1]. The investigation in biomechanics of tactile perception may help us to understand the mechanism of tactile sensation.

Two categories of fingertip models have been proposed on the tactile discrimination of one or two-point tactile stimuli. One category is the structural model, which refers to linear elastic half-space model and the multilayer FE model [2]. The other is the physical model constructed based on the quasi-viscoelastic constitutive equation [3]. [4] developed an elastic half-space model based on standard continuum mechanics theory. It was assumed that for small deformations the mechanical properties of skin were adequately approximated by a homogeneous, elastic, and isotropic medium. However, the model, without considering the multi-layers of skin, could not describe the mechanics property of fingertip exactly under loading. [5] built a 'waterbed' model on the basis of [4], however, it did not take the skin thickness into account. Based on the waterbed model, when skin was forced by a line load, the surface deformation did not agree well with the real skin. [6] constructed a finger model considering the material heterogeneity and geometric structure, and used the finite deflection theory to adapt the large deformation under loading. This model could simulate the surface deflection profiles of finger under load, but it was only suitable for the applications of the given load. An FE model based on anatomical structure of fingertip may potentially overcome those limitations mentioned above. [7] developed an FE model to analyze the influence of the complexity and the fine structure of finger skin (such as epidermal ridges and papillate) on the deformation of the soft tissue inside the fingertip. [8] built an FE model to study the injury of soft tissue and bone in the fingertip arising from the operating work. The results demonstrated that it was feasible for the FE model to simulate the response of soft tissues to loading. In most of the FE models, the skin and subcutaneous tissue layer were regarded as being linearly elastic, and the analyzed data got along well with the experimental data under the condition of small deformation. However, the skin layers and the subcutaneous tissues are nonlinear and time-dependent [9, $10,11]$. The response to the mechanical stimulation should also be nonlinear and time-dependent correspondingly. It is therefore inadequate to consider the linear condition only. It is well known skin is a multilayer structure including epidermis, dermis, subcutaneous tissue, bone and nail. There are different types of mechanoreceptors in each layer. In literatures of $[2,3,12]$, 
they showed that the epidermis was of hyper-elastic behavior and the dermal tissues were of linearly viscoelastic behavior when skin experienced large deformation. [13] tested that the mechanical behavior of the skin and the subcutaneous tissue can be simulated using the nonlinear biphasic constitutive model. [11] proposed a large deformation biomechanical model for pressure ulcers. [14] defined the fingertip skin layers as hyper-elastic and viscoelastic and considered subcutaneous tissue as a porous elastic material, and developed a structural model based on the definition of different tissue materials. The validity of the model was tested with analyzing the skin biomechanics under a line load. However, when solving complex contact problems, it was prone to be of non-convergence and low efficiency.

\section{The Fingertip Finite Element Model}

In this study, we take into account the following aspects:1) The epidermis is considered to be linear elastic under a small deflection for its thinness. The Young's moduli of the epidermis, the bone and nail are assumed, according to the published experimental data [15], to be $0.072 \mathrm{MPa}$, 17.0 GPa and 170.0 MPa, respectively; while Poisson's ratio is assumed to be 0.30 for them. 2) For the deformation parameters of dermis and subcutaneous tissue, the stress vector are the sum of elastic stress vector and viscous stress vector, and the parameters of the soft tissue material model are based on $[4,11,16]$.

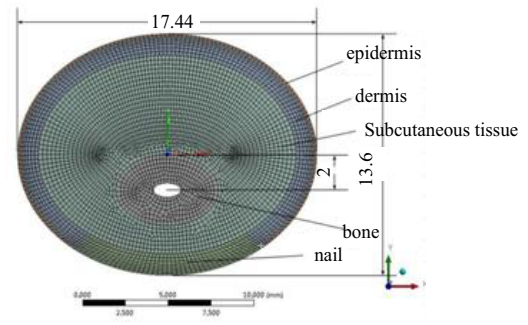

Figure 1. The proposed fingertip FE model.

A cross-section of two-dimensional FE model for the index fingertip is shown in Figure 1. The contour of a fingertip is measured using a reading microscope [8]. The geometry of the fingertip profile is reduced to be an ellipse with a long axis of $17.44 \mathrm{~mm}$ and a short axis of $13.06 \mathrm{~mm}$. The hollow bone is simplified as an ellipse too. From the center of the hollow bone to the center of the outline, the distance is $2 \mathrm{~mm}$ in the $\mathrm{Y}$ direction and $0 \mathrm{~mm}$ in the $\mathrm{X}$ direction. Each layer has the axisymmetric tissue in the $\mathrm{X}$ direction. The thicknesses of the epidermis, dermis, and nail layer are assumed to be $0.2 \mathrm{~mm}, 1.0 \mathrm{~mm}$ and $1.2 \mathrm{~mm}$ respectively.

This model is developed based on the universal software of ANSYS Workbench. The model is meshed using the hexahedron unit, with a size of $0.1 \mathrm{~mm}$ on each side. Considering the fact that the discrimination threshold of the human finger is $2 \mathrm{~mm}$ [17], the model has a high resolution. And we can infer that the depth of the model is only $0.1 \mathrm{~mm}$ too. Hence it can be approximated as a $2 \mathrm{D}$ model. In the simulation, it is assumed that there is no relative sliding and separation between any two adjacent layers, and the degree of freedom (DOF), the motion law and the biomechanical responses of all points at the connection surface are consistent.

Since the model is symmetric along the X-axis, we only choose the right half as the analysis object. During the practical operation, the contact displacement produced by the load on skin is always less than $2.0 \mathrm{~mm}$. Therefore epidermis can be considered as linearly elastic. Both dermis and subcutaneous tissues are hyper-elastic and linearly viscoelastic materials. The constitutive equations of those materials are referenced from [18]. The bone and the nail are considered as a linearly elastic material with a large Young's modulus. The parameters of epidermis, bone and nail are based on $[7,8]$.

\section{The Fingertip Finite Element Model Simulation}

In this section, we will simulate the tactile biomechanics of skin under distributed load and sharp wedge load stimulation. In biomechanics, the following relations are expected to be found: the relation of the external pressure with the skin displacement, the distribution of pressure in the fingertip under distributed load, and the displacement of the fingertip surface under sharp wedge load. In electrophysiology, we will examine the relation between the biomechanical responses of the subcutaneous tissue stimulated by a T-shaped tool and the discharged rate evoked by the slowly adapting type I sensors (SA I ).

\subsection{The distributed-load stimulation experiment}

Assuming that the displacement $\delta$ of the distributed load (Figure 2) is $2.0 \mathrm{~mm}, 2.34 \mathrm{~mm}, 2.5 \mathrm{~mm}$ and $2.8 \mathrm{~mm}$, which are consistent with $[2,3,19]$, the contact force of fingertip under a distributed load at different distance is determined and compared with the experimental measurements of $\mathrm{Wu}$ and Jindrich as shown in Figure 3.

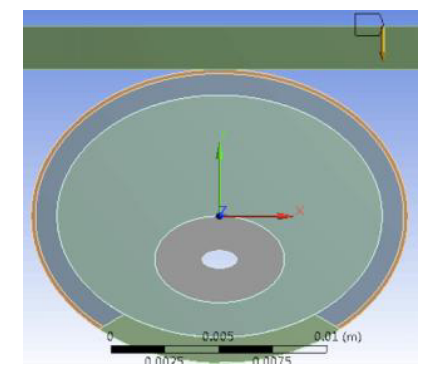

Figure 2. Distributed load applying to the skin surface.

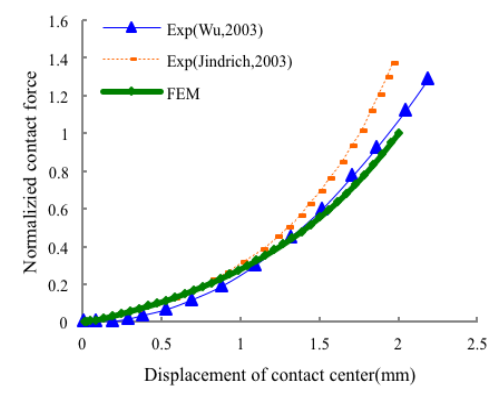

Figure 3. Comparison of predicted contact forces with $[2,3]$. 
From the figure 3, we can see that when the load is $40 \%$ of the maximum force, the size of deformation is up to $80 \%$ of the maximum value. With the increase of the pressure, the rate of change decreases gradually, which is identical to those of $[2,3]$.

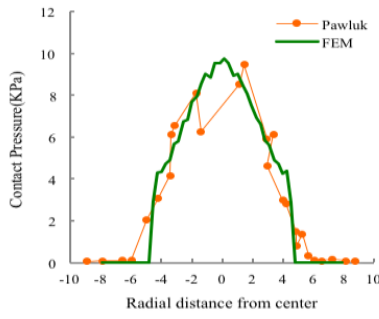

(a)

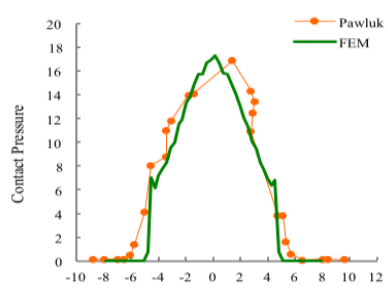

(b)

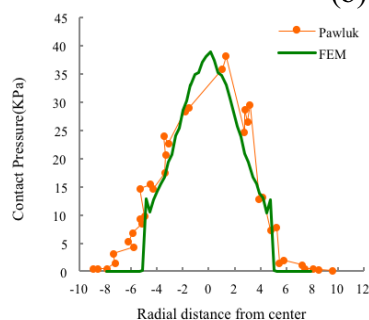

(c)

Figure 4. The predicted distributions of contact pressure under a $\delta=2.34 \mathrm{~mm}, \mathbf{b} \delta=2.5 \mathrm{~mm}$ and $\mathbf{c} \delta=2.8 \mathrm{~mm}$.

The difference of distributed load may influence the distribution of contact pressure in the direct vicinity of the contact center as shown in Figure 4. The profile between the curves produced by FEM and reported by [19] are almost identical. We also can find that the curves of FEM are entirely symmetric while those of Pawluk and Howe's are right-oblique, i.e. the peak value of contact pressure of the experiments occurs at the point of $X=2 \mathrm{~mm}$, not the point of $X=0 \mathrm{~mm}$. This may be due to the fact that in the FEM, the index finger is assumed to be idealized symmetric.

\subsection{The sharp wedge stimulation experiment}

The sharp wedge is considered to be rigid, with the width of $0.05 \mathrm{~mm}$ and $1.0 \mathrm{~mm}$ on the tip and base respectively, and a height of $2.0 \mathrm{~mm}$. Applying the tip end on fingertip, when the displacement constraint is kept to $1 \mathrm{~mm}$, the contact model of finger is developed as Figure 5. After simulation with this model, the skin deformation is plotted in Figure 6.

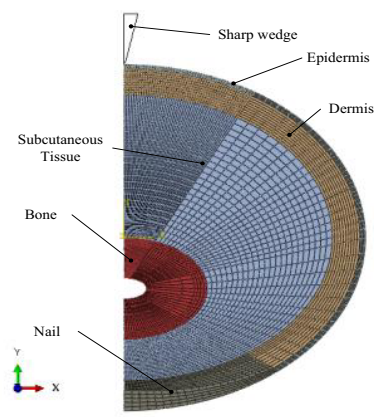

Figure 5. The contact model of fingertip under the sharp wedge load.

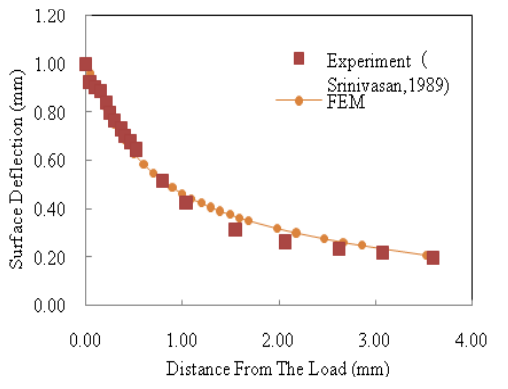

Figure 6. Comparison of predicted deformation with [20].

As Figure 6 shows the two curves match well in the all profiles. The farther the distance of the skin from the stimulated center, the less the deformation, and the rate of reduction decreases with the increase of the distance. When the distance is over $3 \mathrm{~mm}$, the decrement becomes almost negligible.

Literatures[12,21,22,23] suggested that the evoked discharge rate could be explained by SED. Figure 7 depicts the predicted distribution of the SED within the tissues.

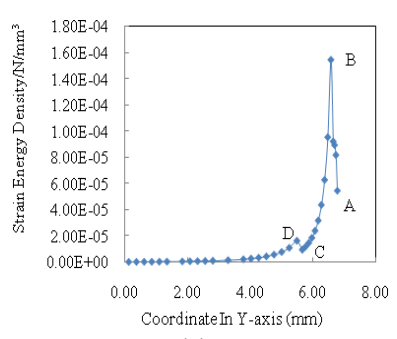

(a)

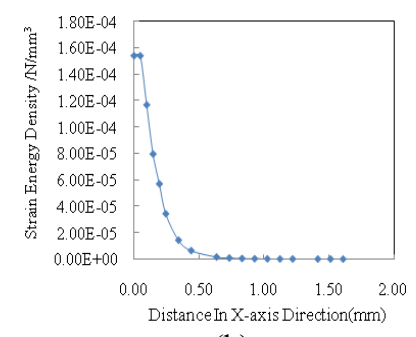

(b)
Figure 7. The predicted distribution of the SED within the tissues. a In the Y direction, $\mathbf{b}$ in the $\mathrm{X}$ direction.

In Figure 7(a), the letters A and B represent two different points located in the connection area between the epidemics and the dermis, at the depth of $6.7782 \mathrm{~mm}$ and $6.57487 \mathrm{~mm}$ from the un-deformed skin surface respectively. The letters $\mathrm{C}$ and $\mathrm{D}$ are two different points, both at the depth of about $5.5 \mathrm{~mm}$ which are the connection area between the dermis and the subcutaneous tissue. As shown in the curve, B and D are two positive peak values of the SED, which just belong to different skin layers. In particular, B is the maximum value point of the curve. It is interesting to note that the SED of tissue presents a monotonous change in the magnitude within the same layer, while there is a turning point $\mathrm{B}$ in the magnitude of SED from one layer to the next one. The simulation results also indicate that the strain energy mainly accumulates in the range between $\mathrm{A}$ and $\mathrm{D}$, where most of the mechanoreceptors are located. The predicted horizontal distribution of the SED of the tissue between the dermis and the subcutaneous is illustrated in Figure 7(b). It shows that at a given depth the tissues of different distance from the contact center present different horizontal SED. It is well known that different shape of the load will produce different strength sensed by the activated receptors and may produce different horizontal SED within tissues. In most cases, the profile of the horizontal SED can map the shape of the stimulator and may help us to obtain the outline of the stimulator. 
When the displacement of the sharp wedge load is $1 \mathrm{~mm}$, the stress distribution within the skin tissue is also simulated as illustrated in figure 8 . The predicted vertical stress and the horizontal stress are presented in figure 8(a-b) respectively. A comparison of figure 8(a) with 7(a) suggests that the predicted distribution of vertical stress agrees well with that of vertical SED. So does a comparison of figure 8(b) with 7(b). Figure 8(c) shows the predicted distribution of the Equivalent Von Mises stress, and we can see the deformation is not continuous.

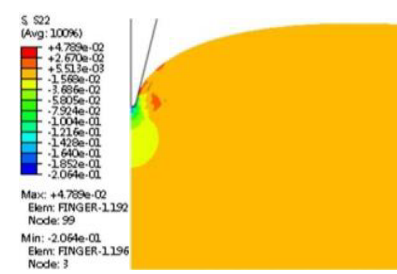

(a)

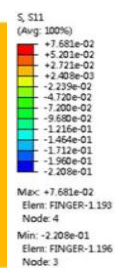

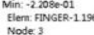

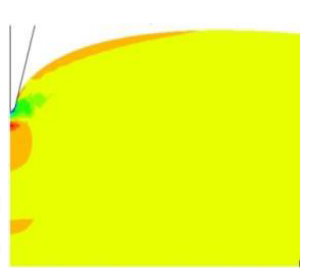

(b)

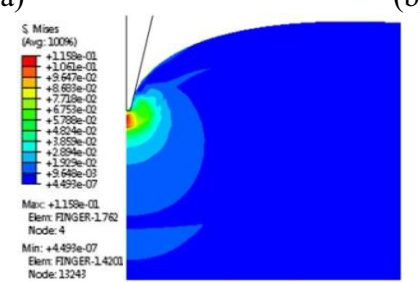

(c)

Figure 8. The predicted stress distributions. a the vertical stress, b the horizontal stress, $\mathbf{c}$ the equivalent von Mises stress.

The distributions of the maximum and minimum normal strains, shear strain, and strain energy density at depths of $0.20,0.70$, and $1.20 \mathrm{~mm}$ from the undeformed skin surface are derived over the width of the fingertip, and are shown in Figure 9(a-d), respectively, where the values are all represented by their logarithms.

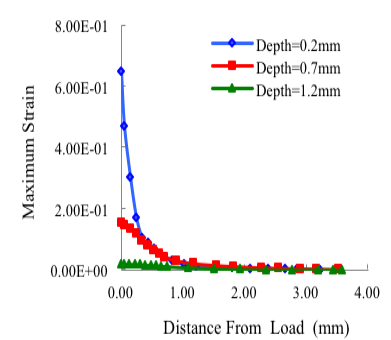

(a)

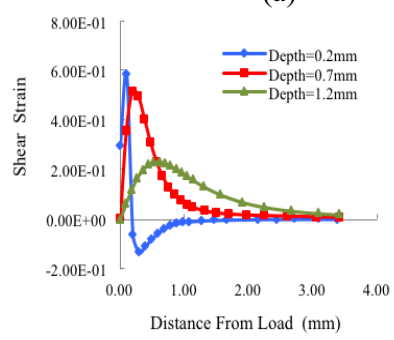

(b)

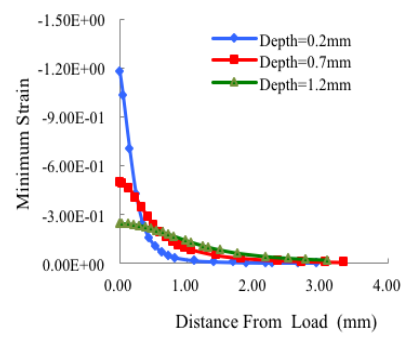

(b)

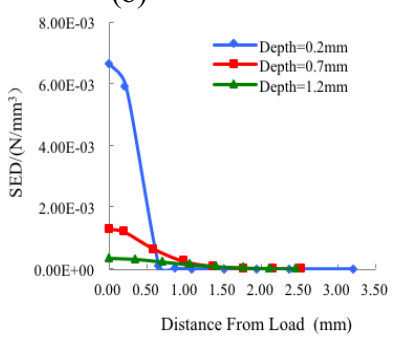

(d)
Figure 9. Predicted distributions of the fingertip indented using a sharp wedge (at depths of $0.20,0.70,1.20 \mathrm{~mm}$ ). a The tensile strain, $\mathbf{b}$ the compressive strain, $\mathbf{c}$ the Shear strain, $\mathbf{d}$ the SED.

The maximum (tensile) strain of the tissue predominantly occurs in a direction parallel to the skin surface, while the minimum (compressive) strain mostly occurs in a direction normal to the skin surface. The maximum and minimum strain and the SED exhibit their maximal magnitudes in the immediate vicinity of the contact point, while the shear strain reverses its direction around the contact point.

From Figures 7-9, we may find that when skin is stimulated by a sharp wedge, the stress/strain of skin show a high gradient at the junctional plane between two layers. As we know, the mechanoreceptors are mainly located in the dermis layer, and very sensitive to the internal strain of the skin tissues. Therefore it makes sense to research the tactile sensation of the fingertip through investigating the strain distribution.

\subsection{The electrophysiological experiment}

During the simulation, it is assumed that: 1) The most sensitive point (MSP) of contact section is the center of the skin surface. 2) The deformation of soft tissue at a given depth represents the sensing intensity.

Based on the analysis above, it is known that these two assumptions are rational. In this experiment, a Tshaped hard metal tool with the convex width of $3.0 \mathrm{~mm}$ or $1.0 \mathrm{~mm}$ and the edge chamfer radius of $0.1 \mathrm{~mm}$ is served as the stimulator. The friction coefficient of $\mathrm{T}$-shaped tool and skin surface is set as 0.3 according to [18].

The procedures of the experiment are as follows: 1) From the start point of the left edge through the MSP to the end point of the right edge, an action route parallel to the $\mathrm{X}$-axis is determined. 2) At any action point, within $0.005 \mathrm{~s}$, the T-shaped tool is pressed vertically to $1.0 \mathrm{~mm}$ deep into skin at a constant speed, and then it is withdrawn to the starting point at the same speed. 3) After that, it is moved to the right at a step of $0.2 \mathrm{~mm}$. And then, it will repeat the operation of 'press-withdrawmove right-press'. This is repeated until it reaches the end point on the right edge.

Figure 10(a) provides a comparison of the predicted SED of soft tissue at some given depths with the discharge rate of SA I, where the dotted line represents the discharge rate reported by [4], and the diamond line and triangle line illustrate the SED of soft tissue at $1.0 \mathrm{~mm}$ and $0.75 \mathrm{~mm}$ respectively. There are some deviations between the discharge rate and the two SEDs. In comparison, the one at $0.75 \mathrm{~mm}$ matches better with the discharge rate than the one at $1.0 \mathrm{~mm}$. It indicates that the matching rate is in terms of the depth where the receptor locates. However, the existing deviation between them may result from the differences of the mechanical and geometric properties for the involved biological soft tissue. A comparison of the predicted maximum tensile strain (Ten), minimum compressive strain (Com), SED of soft tissue and the mean discharge rate at the depth of $0.75 \mathrm{~mm}$ is drawn in Figure 10(b). The SED presents the high conformity with the discharge rate. The correlation coefficient between them is 0.94 , which is more than that of other two mechanical responses with the discharge rate. 


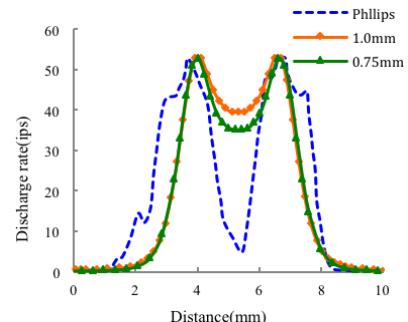

(a)

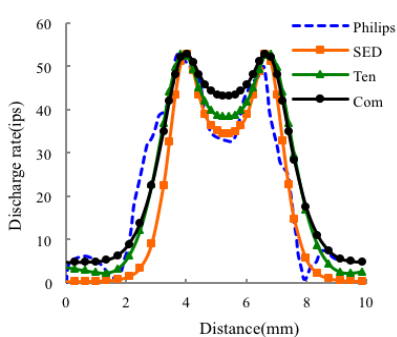

(b)
Figure 10. A comparison between the simulated mechanical responses and the discharge rate reported by [4] when the bar width of the T-shaped block is $3.0 \mathrm{~mm}$. a The predicted SED at the depth of $1.0 \mathrm{~mm}$ and $0.75 \mathrm{~mm}, \mathbf{b}$ The predicted SED, the Ten, the Com of tissues at the depth of $0.75 \mathrm{~mm}$.

To observe the effect of bar width onthe match between the mechanical responses and the discharge rate, we also conduct the same simulation when the bar width of the T-shaped tool is $1.0 \mathrm{~mm}$, as shown in Figure 11 . Compared with the Figure 10, it presents a better consistency between the simulation results and the data in literatures. Obviously, their deviations decrease with the reduction of the bar width. In addition, the predicted SED at the depth of $0.75 \mathrm{~mm}$ still fits better with the public data than that at the depth of $1.0 \mathrm{~mm}$, which is identical to the condition of $3.0 \mathrm{~mm}$ bar width.

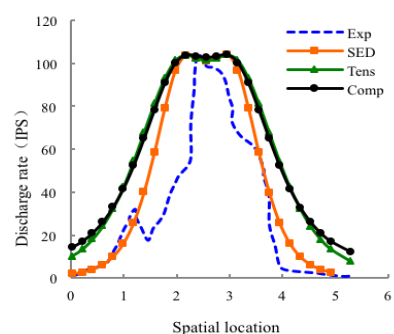

(a)

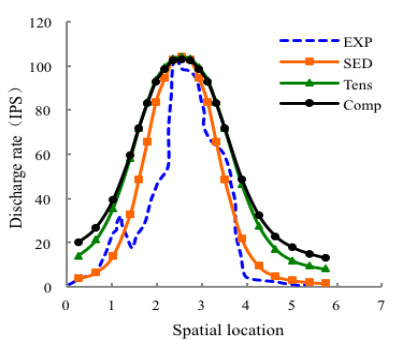

(b)
Figure 11. A comparison of the predicted SED, the Ten, the Com of tissues at two different depths with the discharge rate reported by [4] when the bar width of the T-shaped block is $1.0 \mathrm{~mm}$. a Depth $=0.75 \mathrm{~mm}$. b Depth $=1.0 \mathrm{~mm}$.

\section{Conclusions}

This paper presented a 2D FE model for fingertip skin to investigate the relationship between the deformation and the sensation. By comparing predicted mechanical responses using the proposed model with the results reported by literatures, they match well in the overall profile, the value of the key points, the changing tendency, and so on. All of the above can illustrate the validity of the model. In addition, the simulation results show that the soft tissues of the fingertips are very sensitive to the external stimulus, and the predicted distribution characteristics of the SED within soft tissues can map the evoked charging rate of mechanoreceptors effectively. Therefore, the model is examined to be valid and can be applied in the tactile research.

\section{Acknowledgements}

This work was supported by the National Natural Science Foundation of China (61175069), the Natural Science Foundation of Jiangsu Province of China (BK20131205) and 2012 Qing Lan Project of Colleges and Universities of Jiangsu Province.

\section{References}

1. M.A. Srinivasan, R.H. LaMotte, J. Neurosci. 7, 6: 1682-1697 (1987)

2. D.L. Jindrich, Y.T. Zhou, J.T. Dennerlein, J Biomech. 36, 4: 497-503 (2003)

3. J.Z. Wu, R.G. Dong, W.P. Smutz et al, Med. Eng. Phys. 25, 3: 397-406 (2003)

4. J.R. Phillips, K.O. Johnson, J. Neurophysiol. 46, 6: 1192-1203 (1981)

5. M.A. Srinivasan, K. Dandekar, J. Biomech. Eng. 118, 1: 48-55 (1996)

6. E.R. Serina, E. Mockensturm, C.J. Mote, D. Repmel, Journal of biomechanics, 31, 7: 639-646(1998)

7. T. Maeno, K. Kobayashi, N. Yamazaki. JSME 41, 1: 94-100 (1998)

8. J.Z. Wu, R.G. Dong, Proc. IMechE. 219, 9: 89-103 (2005)

9. Y.C. Fung, S.C. Cowin, Springer-Verlag, 2nd ed. New York (1994)

10. M.B. Rubin, S.R. Bodner, N.S. Binur, ASME J Biomech Eng, 120, 5: 686-9 (1998)

11. Y. Zheng, A.F. Mak, Biomedical Engineering IEEE Transactions on, 43, 9: 912-918 (1996)

12. A.B. Vallbo, R.S. Johansson, Hum. Neurobio. 3, 1: 3-14 (1984)

13. V.C. Mow, S.C. Kuei, W.M. Lai et al, ASME J. Biomech. Eng. 102, 1:73-84 (1980)

14. J.Z. Wu, R.G. Dong, S. Rakheja et al, Med. Eng. Phys. 26, 2: 165-175 (2004)

15. H. Yamada, F.G. Evans (1971). The Williams and Wilkens Company, Baltimore, 4(2): 297+x

16. L.H. Pan, L. Zan, F. Foster. Ustrasound Med. Biol. 24, 7: 995-1007 (1998)

17. K.B. Shimoga, IEEE Virtual Reality Annual International Symposium : 271-279 (1993)

18. J.Z. Wu, R.G. Dong, D.E. Welcome, Med. Eng. Phys. 28, (8): 816-826 (2006)

19. D.T. Pawluk, R.D. Howe, Biomech. Eng. 121, 2: 178-183 (1999)

20. M.A. Srinivasan, J. Biomech. 22, 4: 343-349 (1989)

21. K. Dandekar, B.I. Raju, M.A. Srinivasan, J Biomech Eng. 125, 5: 682-691 (2003)

22. K.O. Johnson, Curr. Opin. Neurobiol. 11, 4: 455-461 (2001)

23. K.O. Johnson, T. Yoshioka, F. Vega-Bermudez, J. Clin. Neurophysiol. 17, 6: 539-558 (2000) 